Originally published as:

Korte, M., Constable, C. (2005): The geomagnetic dipole moment over the last 7000 years new results from a global model. - EARTH AND PLANETARY SCIENCE LETTERS, 236, 1-2, 348-358,

DOI: 10.1016/j.epsl.2004.12.031 


\section{The geomagnetic dipole moment over the last 7000 years - new results from a global model}

M. Korte ${ }^{1 *}$ and C. G. Constable ${ }^{2}$

${ }^{1}$ GeoForschungsZentrum Potsdam, Telegrafenberg, 14473 Potsdam, Germany

${ }^{2}$ Institute of Geophysics and Planetary Physics, Scripps Institution of Oceanography, La Jolla, CA 92093-0225, USA

* Corresponding author: Tel: +49 331288 1268, Fax: +49 331 2881235

email: monika@gfz-potsdam.de 


\section{Introduction}

Global spherical harmonic magnetic field models like GUFM (1), covering the whole time span from almost the first direct field observations 400 years ago to 1990, or the International Geomagnetic Reference Field (IGRF, see http://www.ngdc.noaa.gov/IAGA/ vmod/igrf.html) show that Earth's dipole moment has decreased by almost $10 \%$ since Gauss' first analysis in 1830. Archaeomagnetic intensity results have been used to determine the variation of the dipole moment for earlier times than the few centuries for which direct field observations are available. These determinations, however, are approximations of the real dipole moment under the assumption that the field originates only from a geocentric dipole or a geocentric axial dipole. The results are called virtual dipole moment (VDM) and virtual axial dipole moment (VADM) respectively. Because of non-dipole field contributions results from different regions can differ significantly (2). Averaging global results and/or averaging over several hundred years is usually assumed to average out non-dipolar influence. McElhinny and Senanayake (3) determined the VADM variation for the past 50000 years. Yang et al. (4) repeated the study for the last 12000 years with an updated data set of almost three times as many data. Recently, Genevey et al. (5) compiled and critically evaluated another global intensity data set spanning the past 10000 years mainly for the purpose of global field modelling, but also to study VADM results. The different results agree well for some parts of the overlapping time span but show significant differences for others.

Recently the first continuous global spherical harmonic field models based on archaeo- and paleomagnetic data including the absolute intensity compilation by Genevey et al. (5) have been developed (6). CALS7K.2 (Continuous model of Archaeomagnetic and Lake Sediment data of $7 \mathrm{k}$ years) covers the past 7000 years from $5000 \mathrm{BC}$ to $1950 \mathrm{AD}$. The dipole moment for this model is systematically and significantly lower than the above mentioned 


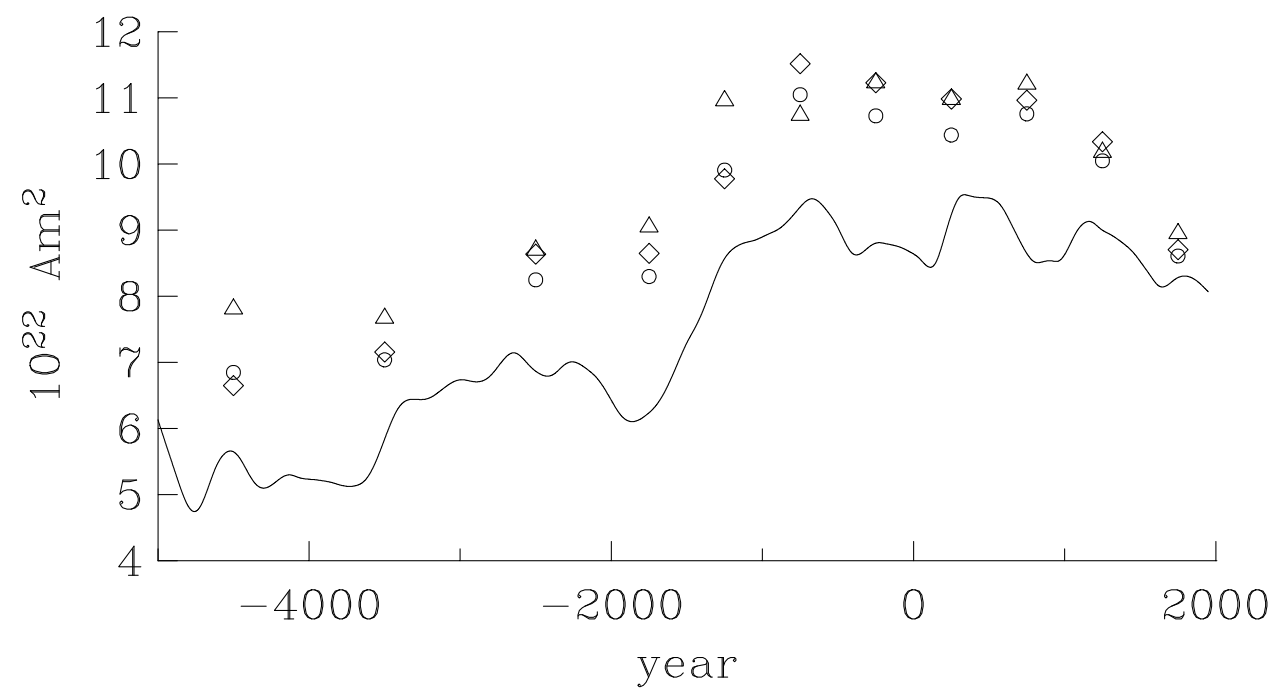

Fig. 1. Comparison of dipole moments: SHA dipole moment of CALS7K.2 (-), averaged VADMs from intensity data on which CALS7K.2 is based $(\circ)$ and previous results from McElhinny and Senanayake (3) ( $\diamond)$ and Yang et al. (4) ( $\triangle$ ) respectively.

VADM results, although it shows the same general trends. We investigate the source of these discrepancies which are illustrated in Fig. 1.

We consider several possible explanations related to the quality and distribution of the data, and the influence of non-dipole fields and both temporal and spatial data distribution. The intensity data sets used in earlier VADM compilations may be systematically different from that used for CALS7K.2. Also the data to which the model are fit are predominantly directional measurements while the field strength, i.e. the scaling, is governed by the intensity data. In an ideal case, with infinitely dense and accurate data, and a magnetic field with no more than two poles, the scaling is independent of the directions (7). In practice, fit to intensity data can be improved to a certain degree at the cost of fit to directional data and vice versa. VDMs and VADMs do not take into account the non-dipole contributions to the field. Temporal and spatial averaging are used in an attempt to cancel out these contributions. It seems unlikely, however, that they completely cancel over time spans of a few centuries, especially considering the very uneven global distribution of data. Consequently VDMs and VADMs may be too high compared to dipole 
moments obtained by spherical harmonic analysis (SHA).

We use the CALS7K.2 model along with the intensity data set from which it was derived to address the question whether VADMs/VDMs and SHA dipole moment are quantitatively comparable. The influence of data distribution on VDM/VADM results is investigated and implications for studies of dipole moment variation are discussed.

\section{Determination of Dipole moment, VDM and VADM}

We have to distinguish among three different dipole moments, which are commonly determined in the following ways. In spherical harmonic analysis (SHA) global field observations are fit by a series of multipole Gauss coefficients. The first three coefficients, $g_{1}^{0}, g_{1}^{1}$ and $h_{1}^{1}$ describe the contribution of a geocentric dipole. This dipole may be tilted about the rotation axis with $g_{1}^{0}$ being the axial contribution. In SHA models from recent data the contribution from an axial dipole tilted by approximately $11^{\circ}$ accounts for more than $98 \%$ of the geomagnetic field in terms of power at the Earth's surface. The magnetic dipole moment $M$ is given by

$$
M=\frac{4 \pi}{\mu_{0}} R^{3} \sqrt{\left(g_{1}^{0}\right)^{2}+\left(g_{1}^{1}\right)^{2}+\left(h_{1}^{1}\right)^{2}}
$$

with $R=6371.2 \mathrm{~km}$ the average radius of the Earth and $\mu_{0}=$ $4 \pi \cdot 10^{-7} \mathrm{Vs} /(\mathrm{Am})$ the permeability of free space.

In paleomagnetism, VDM and VADM are determined from paleointensity results (e.g. 8). For an intensity measurement $F$ a dipole moment $P$ is obtained by

$$
P=\frac{4 \pi}{\mu_{0}} R^{3} \frac{F}{\sqrt{1+3 \cos ^{2} \theta}} .
$$

If $\theta$ is geomagnetic colatitude, then $P$ is the VDM, the magnetic moment of a geocentric dipole. If $\theta$ is geographic colatitude, then 
$P$ is the VADM, the magnetic moment of an axial geocentric dipole. In either case contributions of higher degree and order cannot be taken into account. The presence of such contributions in the geomagnetic field thus leads to deviations of VDM and VADM from the actual geocentric or axial geocentric dipole moment. The practical difference between VDM and VADM arises from the fact that the geomagnetic colatitude $\theta$ can be determined if inclination $I$ is known through

$$
\tan I=2 \cot \theta .
$$

Inclination information, however, is often not available along with intensity results. Then only VADMs can be determined. While Yang et al. (4) calculated solely VADMs, McElhinny and Senanayake (3) used VDMs where possible and their final results are a combination of about equal numbers of VDMs and VADMs.

\section{Data and characteristics of the global model}

Genevey et al. (5) compiled a new global dataset of archaeointensity data spanning the past 10000 years. For this study we only use data from the past 7000 years because that is the longest extent of a continuous global SHA model (6). For the time-interval between $5000 \mathrm{BC}$ and $1950 \mathrm{AD}$ the number of data is 3188. As in previous VADM studies and discussed in detail by Genevey et al. (5) the global distribution is far from even. Table 1 gives an overview for different regions. Almost half of the data are from the European region. The bulk of the remaining data are from Asia, and from the whole Southern hemisphere there are only 167 values coming from small regions in South America and Australia. The data compilation comes with error estimates. For the global model described below, all data were initially used and a few data $(3 \%)$ were subsequently rejected as outliers due to their large misfit with a preliminary model (see Korte and Constable (6) for details). Note that the rejected data were not necessarily 


\begin{tabular}{lrr}
\hline Region & Total number & Depleted number \\
\hline Europe & 1502 & 1492 \\
Asia & 1265 & 1209 \\
North America & 242 & 226 \\
Southern hemisphere & 167 & 165 \\
Global & 3188 & 3092 \\
\hline Table 1 & &
\end{tabular}

Number of intensity data in different regions.

the least reliable according to their error estimates, confirming that these estimates reflect to a large degree only our knowledge (or lack thereof) about the data rather than the actual quality. The average error estimate is $11 \mu \mathrm{T}$ in both the total and the depleted intensity data sets, which is quite large.

The almost normally distributed residuals have a bias of $3.6 \mu \mathrm{T}$, i.e. model predictions on average are slightly lower than the data. This is not caused by over-regularization, but due to a combination of weighting the data by the uncertainty estimates and the fact that the fit to directional and intensity data is not completely independent, i.e. intensity does not act purely as scaling factor. The measurement uncertainty estimates for intensity are percentage errors, which means that there is a certain correlation between high values and large error estimates so that minimising the root mean square (rms) misfit can introduce a bias in the residuals. The bias, moreover, cannot be reduced by increasing the dipole moment without unreasonably increasing the misfit to the directional data in the modeling, so it must reflect measurement error in the data or non-dipole field structure of higher degree and order than in CALS7K.2.

We will compare results from the full and the depleted intensity data set of 3092 values in this study. Table 1 gives the comparison of numbers per region. Fig. 2 shows the global data distribution for the time intervals used in this study by contouring the logarithm of data concentration by area, in effect a kind of density function for observations. The scale is such that the integral over 


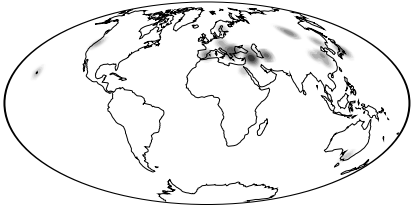

Archeo Int 5000 - 4000 BC

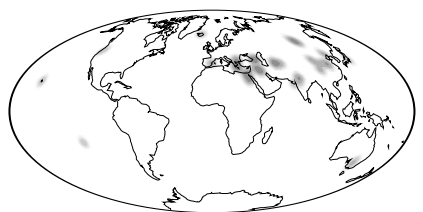

Archeo Int $2000-1500$ BC

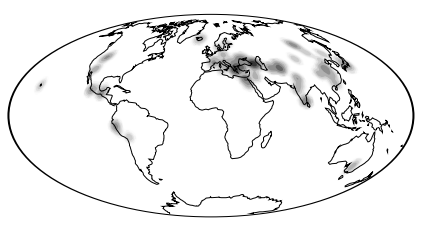

Archeo Int 500 - 0 BC

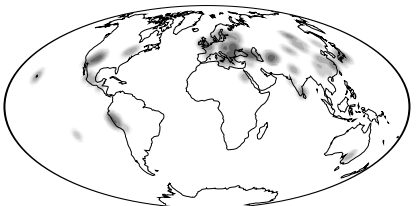

Archeo Int $1000-1500$ AD

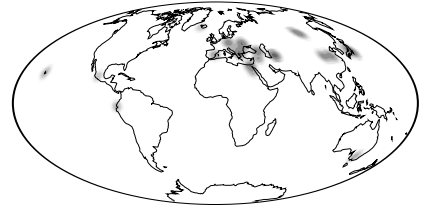

Archeo Int $4000-3000$ BC

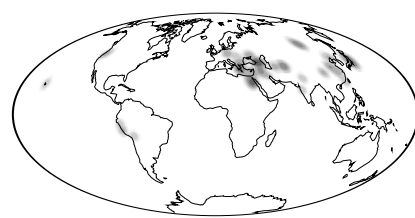

Archeo Int 1500 - 1000 BC

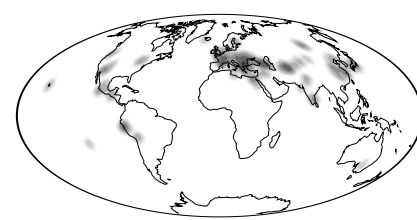

Archeo Int $0-500 A D$

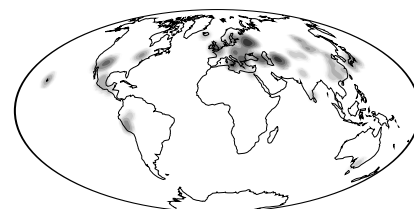

Archeo Int $1500-2000$ AD

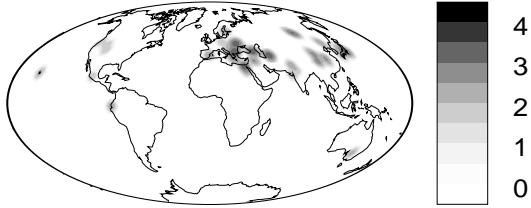

Archeo Int $3000-2000$ BC

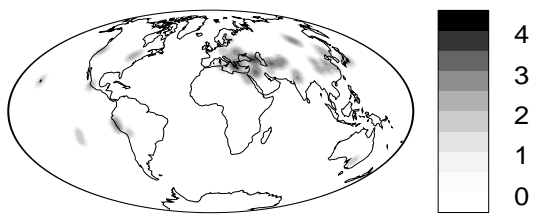

Archeo Int 1000 - 500 BC

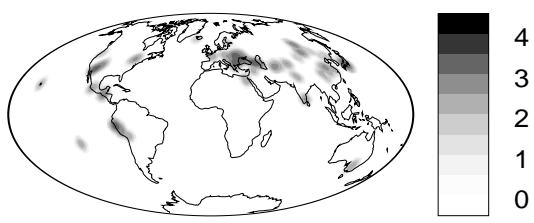

Archeo Int $500-1000$ AD

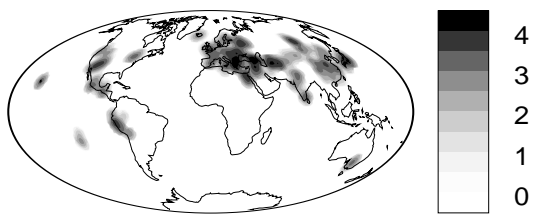

Archeo Int 5000 BC - 2000 AD

Fig. 2. Contoured concentration of intensity data through time for the time intervals used in this study.

the whole Earth's surface returns the total number of data points within the time interval. For details of this computation see Korte et al. (9). The figure is given for the total number of data but can also be taken as representative for the depleted data, as the rejected data are not concentrated in a specific region.

The calculation of VADMs by equation 2 from the intensity data is straightforward and had already been carried out by (5). The averaging intervals were chosen the same as in the former studies by McElhinny and Senanayake (3) and Yang et al. (4), namely 500 years back to $2000 \mathrm{BC}$ and 1000 years for earlier times. The original reason for choosing two different intervals was the fact that fewer data exist for the earlier epochs so that 500 years do not contain enough individual results to give a good average. This is still a valid argument as can be seen from the histogram 


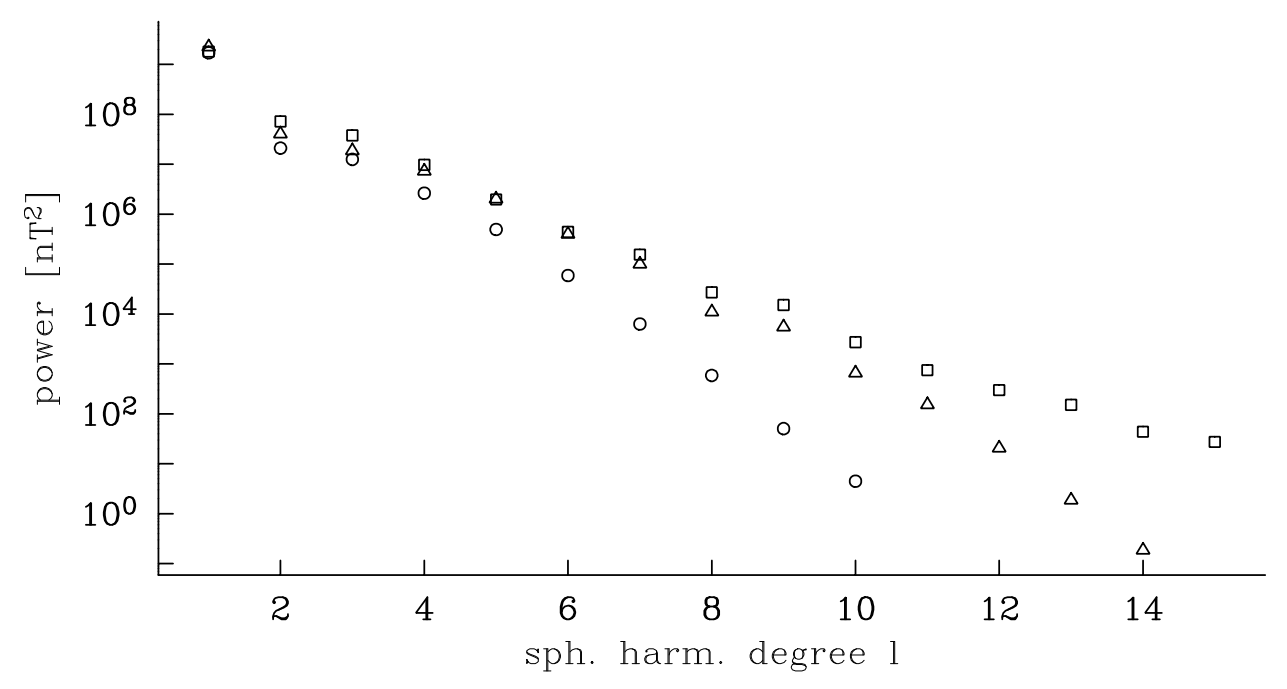

Fig. 3. Geomagnetic field spectrum of archaeomagnetic model CALS7K.2 (o), historical model GUFM $(1)(\triangle)$ and recent satellite model POMME (12) (口). For GUFM and CALS7K.2 spectra over each model's whole time interval were averaged.

of temporal distribution in the paper by Genevey et al. (5).

The global model CALS7K.2 (6) is based on the intensity data compilation and archaeomagnetic and lake sediment declination and inclination data (9). The spatial basis is regularised spherical harmonics up to degree and order 10 of which the first 4 to 5 degrees can be reliably resolved. The temporal continuity is given by cubic B-splines. A comparison of spatial power spectra (Fig.3) shows that compared to recent models with much better resolution but shorter time spans CALS7K.2 has less power in the higher degrees. For our study this implies that differences between dipole moment and VDM/VADM from this model are likely to be underestimated.

The SHA dipole moment was calculated for each year by equation 1, and additionally averaged over the same time intervals as the VADMs. We also calculated intensity predictions from model CALS7K.2 with the same temporal and spatial distribution as the input intensity data, obtaining once 3188 and once 3092 synthetic values. Additionally the model predictions of inclination at those locations were determined. Using equations 2 and 3, VADMs and VDMs were calculated for each point and averaged 


\begin{tabular}{llc}
\hline Name & Explanation & Symbol \\
\hline$M$ & SHA dipole moment from CALS7K.2 & - \\
$A_{d}$ & Virtual axial dipole moment based on real data & $\bullet$ \\
$A_{m}$ & Virtual axial dipole moment based on model predictions & + \\
$D_{m}$ & Virtual dipole moment based on model predictions & $*$ \\
\hline
\end{tabular}

Table 2

Nomenclature used for different kinds of dipole moments in text and figures.

in the same intervals as the results from the real data.

To clarify the distinction among all these different dipole moments, we introduce the following nomenclature: the SHA dipole moment will be referred to as $M$. VADMs will be called $A$, VDMs $D$, with the subscripts $d$ and $m$ when they are based on real data or model predictions respectively. Table 2 defines the different types of dipole moments we will deal with.

\section{Results}

Figure 4a shows $A_{d}(\bullet), A_{m}(+), D_{m}(*)$ and $M$ of the depleted data set, i.e. exactly the same data and locations used in constructing the model. While the $D_{m}(*)$ are slightly, but systematically higher than the $A_{m}(+)$, both the $A_{m}$ and $D_{m}$ almost all lie between $M$ and $A_{d}(\bullet)$. Errors in the mean from the averaging process are about the size of the plotted symbols. Table 3 lists all the values for exact comparison, together with the standard deviations of the distributions. From Fig. 4b we see that differences between results from the depleted and total data set are negligible, a difference in $A_{d}(\bullet)$ is visible only between $1000 \mathrm{BC}$ and $500 \mathrm{AD}$, where a large number of data were rejected. We do not expect a significant difference as the rejected data include a broad range of intensity values and the technique used for rejection only excludes data which are incompatible with surrounding and global data. In the following we will only consider the depleted data set. 


\begin{tabular}{lrrrrrrrr}
\hline Avg. Time & $M$ & $A_{d}$ & $A_{m}$ & $D_{m}$ & $\sigma\left(A_{d}\right)$ & $\sigma\left(A_{m}\right)$ & $\sigma\left(D_{m}\right)$ & Nr. of data \\
\hline-4500.0 & 5.29 & 6.85 & 6.28 & 7.10 & 1.22 & 0.56 & 0.70 & 215 \\
-3500.0 & 5.85 & 7.04 & 6.02 & 6.32 & 1.29 & 0.57 & 0.54 & 146 \\
-2500.0 & 6.87 & 8.21 & 7.49 & 7.85 & 1.63 & 0.69 & 0.81 & 233 \\
-1750.0 & 6.44 & 8.27 & 7.35 & 7.60 & 1.77 & 0.65 & 0.54 & 103 \\
-1250.0 & 8.35 & 9.97 & 9.51 & 9.25 & 1.93 & 0.57 & 0.62 & 157 \\
-750.0 & 9.21 & 11.10 & 10.57 & 10.72 & 2.09 & 0.86 & 0.88 & 238 \\
-250.0 & 8.75 & 10.61 & 9.89 & 10.23 & 2.06 & 1.06 & 0.91 & 338 \\
250.0 & 9.07 & 10.22 & 9.39 & 9.97 & 1.89 & 0.86 & 0.79 & 393 \\
750.0 & 8.85 & 10.74 & 9.78 & 9.81 & 1.86 & 0.90 & 0.82 & 342 \\
1250.0 & 8.90 & 10.05 & 9.35 & 9.51 & 1.68 & 0.60 & 0.55 & 410 \\
1750.0 & 8.25 & 8.55 & 8.18 & 8.27 & 1.37 & 0.64 & 0.55 & 517 \\
binned average & & 9.24 & 8.53 & 8.78 & 1.71 & 0.72 & 0.7 & \\
true average & 7.41 & & & & & & & \\
\hline
\end{tabular}

Table 3

Values of different dipole moments and standard deviation of their distributions $\left(10^{22} \mathrm{Am}^{2}\right)$. All calculations are based on the depleted data set.

The average difference between $M$ and $A_{d}(\bullet)$ is $1.43 \cdot 10^{22} \mathrm{Am}^{2}$ or $19.3 \%$ of the average $M$. It results from several sources. The average difference between $M$ and $A_{m}(+)$ is $0.72 \cdot 10^{22} \mathrm{Am}^{2}$ or $9.7 \%$ with a maximum of $1.36 \cdot 10^{22} \mathrm{Am}^{2}$. This difference must purely be attributed to non-dipole field contributions not averaging out in $A_{m}(+)$. The differences between $A_{d}(\bullet)$ and $A_{m}$ $(+)$, involve both data uncertainties and higher degree structure which is not resolved in the model. It amounts to $0.71 \cdot 10^{22} \mathrm{Am}^{2}$ or $9.6 \%$ of $M$ on average and reflects the bias of the intensity residuals in CALS7K.2: The influence of the bias on the VADMs can roughly be estimated by eq. 2. Evaluating the constants yields the latitude-dependent relation

$$
P=\frac{0.26 \cdot 10^{22}}{\sqrt{1+3 \cos ^{2} \theta}} F
$$

for $\mathrm{P}$ in $\mathrm{Am}^{2}$ and $\mathrm{F}$ in $\mu \mathrm{T}$. The $3.6 \mu \mathrm{T}$ bias thus would cause VADMs predicted from CALS7K.2 $\left(A_{m}\right)$ to be too low by 0.9 . 

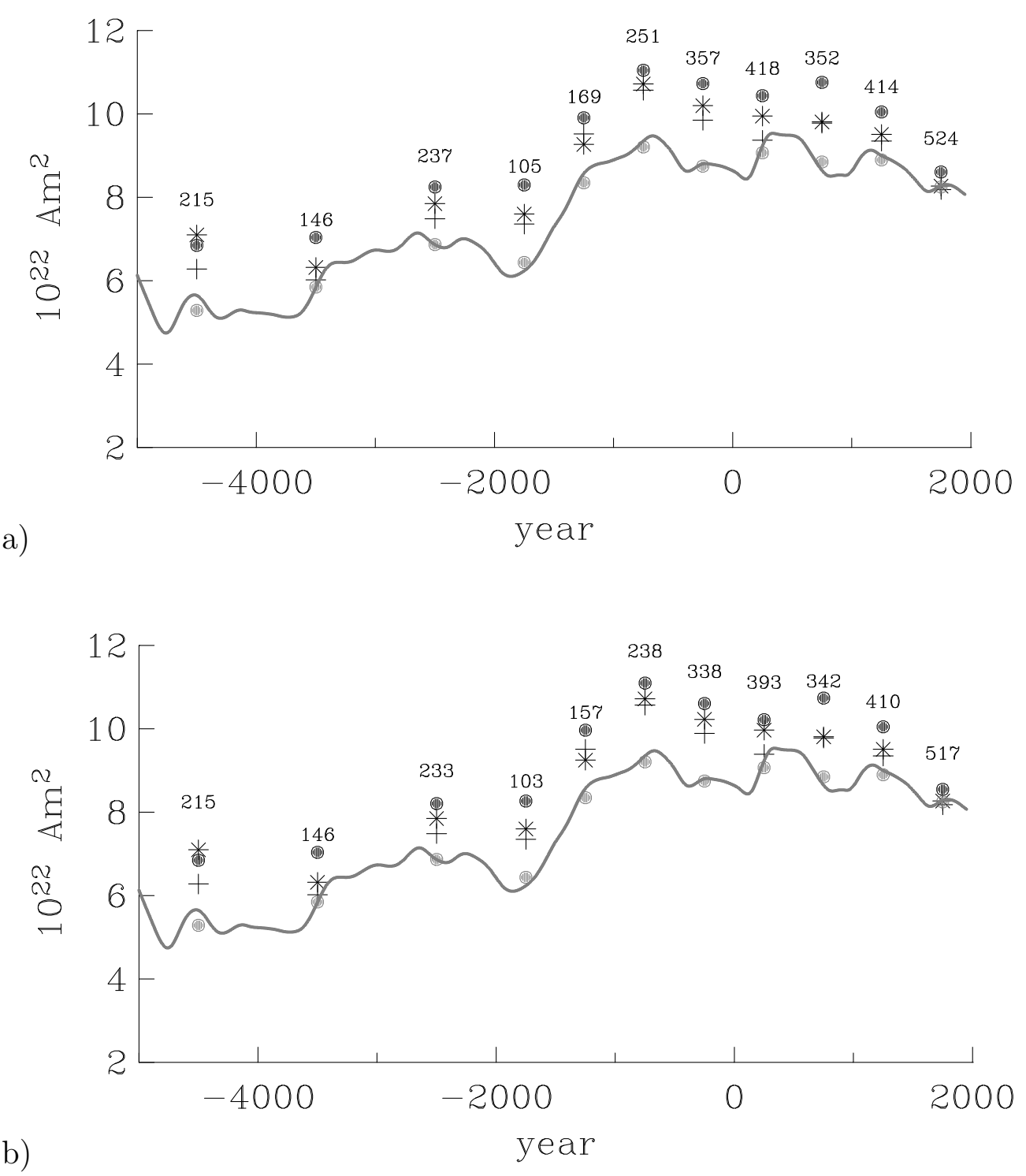

Fig. 4. Comparison of $A_{d}(\bullet), A_{m}(+)$ and $D_{m}(*)$ averaged in 1000 and 500 year bins before and after $2000 \mathrm{BC}$ respectively. $M$ of CALS7K.2 is shown both as continuous gray line and averaged over the same time intervals (gray dots). a) Based on the depleted data, b) based on all 3188 intensity data. The numbers indicate how many data were averaged in each time interval.

$10^{22} \mathrm{Am}^{2}$ if all data came from the equator and half that value if all data came from the poles. This agrees perfectly with the observed average difference as most data come from mid-latitudes.

The standard deviation in the data for the individual averages, both 500 and 1000 year, lies between 16.0 and $21.4 \%$ of the mean for the $A_{d}(\bullet)$, in reasonable agreement with the results obtained by McElhinny and Senanayake (3) (16.3 to $29.4 \%$ ) and Yang 

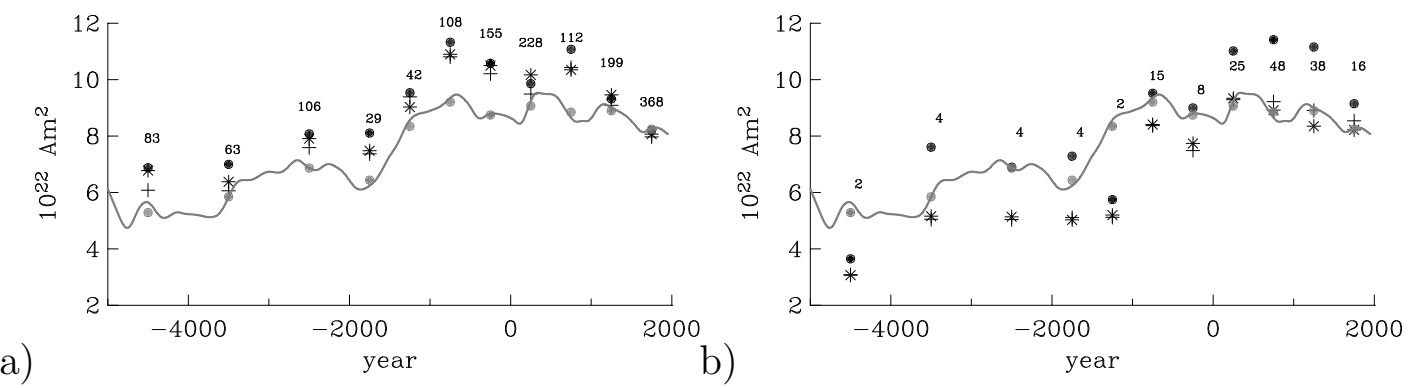

Fig. 5. Comparison of $A_{d}(\bullet), A_{m}(+)$ and $D_{m}(*)$ averaged in 1000 and 500 year bins before and after $2000 \mathrm{BC}$ respectively. a) European region with large numbers of data in every temporal bin (see numbers), b) Southern hemisphere with sparse data coverage. $M$ of CALS7K.2 is shown as the continuous gray line and averaged over the same time intervals (gray dots) in both panels.

et al. (4) (11 to $20 \%$ ). In the undepleted data set this scatter is slightly higher. For $A_{m}(+)$ and $D_{m}(*)$ the standard deviation of the depleted set lie between 5.7 and $10.7 \%$ and between 6.7 and $10.3 \%$ respectively. This difference in VADM scatter between data $A_{d}(\bullet)$ and model predictions $A_{m}(+)$ confirms the conclusion by McElhinny and Senanayake (3) that scatter of the order of 9 to $12 \%$ might be caused by experimental, rock magnetic and dating errors in archaeomagnetic intensity determinations.

Looking for systematic differences in individual regions we observe that $A_{d}(\bullet), A_{m}(+)$ and $D_{m}(*)$ are higher than $M$ everywhere, with only a few exceptions where the number of data is too small for reliable statistics. Two examples from the depleted data set are shown in Fig. 5. We notice that on average there is a closer agreement between $A_{m}(+)$ and $D_{m}(*)$ in the southern hemisphere results than in any northern hemisphere results. This reflects less non-dipolar field structure in the CALS7K.2 model there. We have to be very careful about conclusions from regional differences, however, because we run the risk of circular reasoning. While the temporal averaging of the scarce southern hemisphere data might be too poor to produce the actual average, the global average on the other hand might be strongly biased by the dominance of European and Asian data and might not be the real global average at all. The CALS7K.2 model is based on the same very unevenly distributed data and model predictions therefore might be biased in similar ways. While the 
average model fit to northern and southern hemisphere data is comparable, a significant lack of southern hemisphere non-dipole structure in the model due to sparse data coverage is quite certain, as is the lack of higher order resolution in general compared to models from recent data.

To shed some light on this problem we carried out further comparisons with a regular grid of locations and CALS7K.2 and two recent models with higher resolution. We repeated an analysis of the kind carried out first by Smith (10) and again by Barton et al. (11) and McElhinny and Senanayake (3) based on the IGRFs 1945 and 1975. In these studies large differences in the scatter of VADMs between northern and southern hemisphere were detected. We will study not only the statistics, but latitudinal differences in $\operatorname{VADM}(+)$ and $\operatorname{VDM}(*)$ results themselves. We use a grid with $10^{\circ}$ spacing in latitude and longitude and average the results from circles of latitude from $80^{\circ} \mathrm{S}$ to $80^{\circ} \mathrm{N}$. The two SHA models we use for comparison are POMME (12) and GUFM (1). POMME, based on satellite data with globally equally dense data coverage, is one of the current models with highest spatial resolution. GUFM is the longest continuous model based on direct field observations. Covering 400 years this model allows us to study the success of temporal averaging on almost the right time scale. Note that for the first 240 years GUFM's field strength is constrained by an extrapolation of the axial dipole evolution because there are no intensity measurements and that the spatial resolution increases with time due to increasing number of available data. These are, however, no serious limitations for the following study and we took into account the whole 400 years of GUFM.

Figure 6a confirms the findings of previous IGRF studies, that VADM/VDM determinations and their statistical scatter have strong latitudinal dependency for the recent field. There is no temporal averaging in this case. The low in VADMs/VDMs around $40^{\circ}$ south accompanied by high scatter will be a reflection of the South Atlantic anomaly, which is a regional, non-zonal feature. 

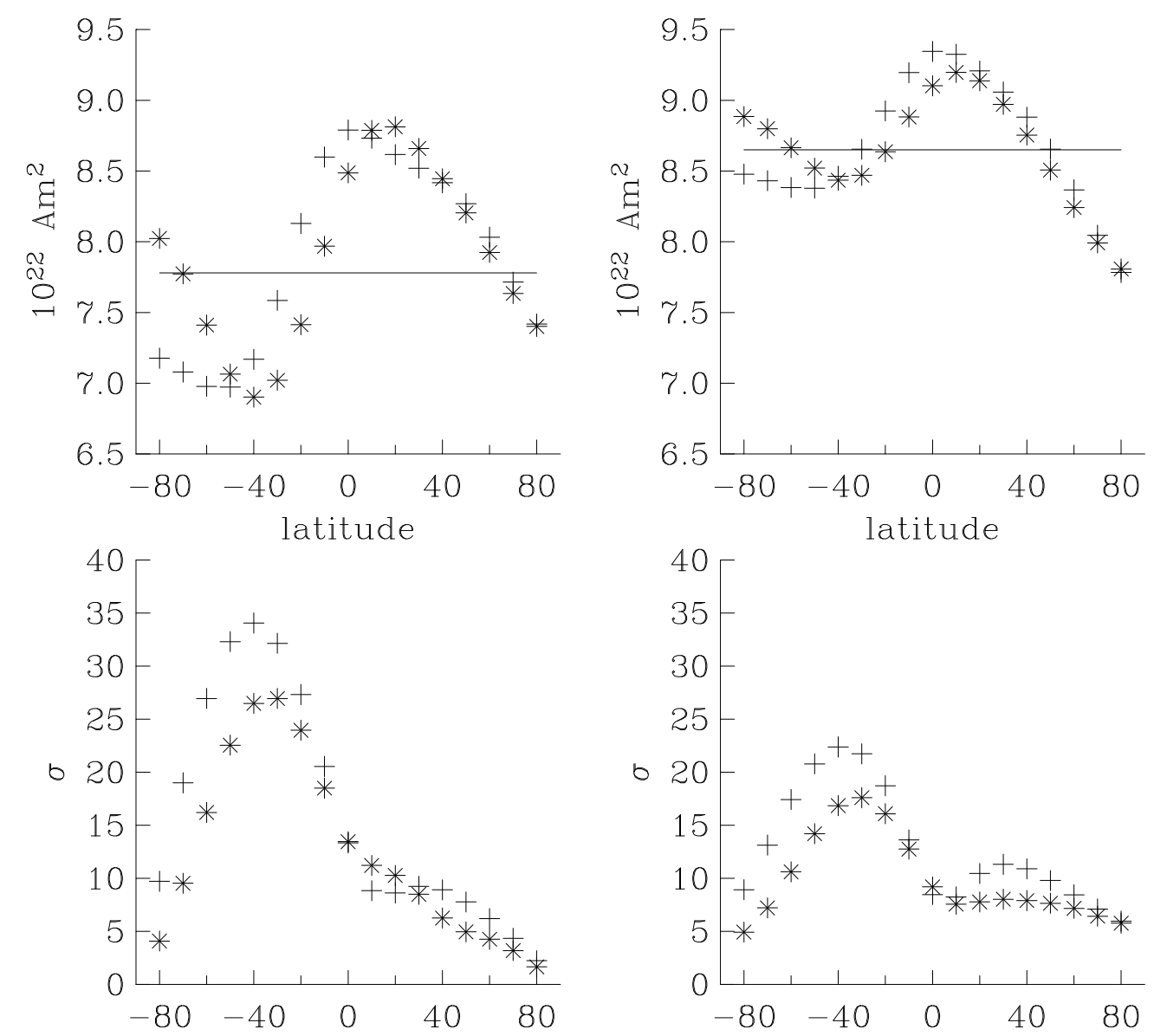

a)

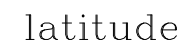

b)

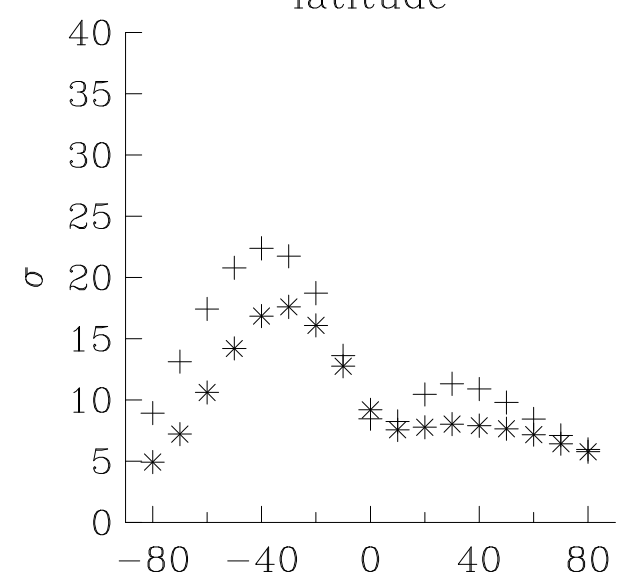

Fig. 6. VADMs $(+)$ and VDMs $(*)$ averaged over latitudinal circles (top) and standard deviation in \% (bottom). The solid line is the SHA dipole moment $M$. a) from POMME model, epoch 2002, b) from GUFM model, average of 1590 to 1990.

The time-scales of non-dipole secular variation might suggest that this present asymmetry is a short-lived phenomenon and latitudinal dependence should decrease with averaging over several hundred years. Figure $6 \mathrm{~b}$, however, shows that 400 years are not enough to completely average out the asymmetry. The results displayed for each latitude here are averages over the whole 400 year time span of the GUFM model of VADMs/VDMs evaluated every 50 years. The global VADM or VDM average is slightly higher than the SHA dipole moment, $M$, for both the POMME and the time-averaged GUFM results. The values are listed in table 4. Our archaeointensity data mainly come from northern latitudes between 10 and $60^{\circ}$. From Fig. 6a we can estimate that for the present field an averaged VADM based on such a data 


\begin{tabular}{lrrrr} 
Model & VADM & VDM & SHA dipole moment & Time interval \\
\hline POMME & 7.89 & 7.87 & 7.78 & 2002 \\
GUFM & 8.68 & 8.65 & 8.65 & $1590-1990$ \\
CALS7K.2 & 7.83 & 7.87 & 7.40 & $5000 \mathrm{BC}-1950 \mathrm{AD}$
\end{tabular}

Table 4

Dipole moments of different models in $10^{22} \mathrm{Am}^{2}$. VADM and VDM are regular grid averages, also temporally averaged for GUFM and CALS7K.2.

distribution would be too high by about $0.5 \cdot 10^{22} \mathrm{Am}^{2}$. For the past 400 years this effect still amounts to 0.1 to $0.2 \cdot 10^{22} \mathrm{Am}^{2}$. For CALS7K.2, the discrepancy between globally and temporally averaged VADM and average SHA dipole moment $M$ is higher again: $0.43 \cdot 10^{22} \mathrm{Am}^{2}$ as listed in table 4 . An asymmetry between the hemispheres with higher averages in the north is present over the whole 7000 years. Two representative time periods are shown in Fig. 7. For this analysis we averaged the VADMs in time over the same intervals as used previously, 1000 years prior to $2000 \mathrm{BC}$ and 500 years for the most recent times. Individual values were calculated every 100 and 50 years respectively. It is obvious again that a data set largely biased towards the northern hemisphere will result in averaged VADMs higher than the global average. In most cases VDMs will give even higher results.

The scatter is lower in GUFM than in POMME (Fig. 6), reflecting the lower spatial resolution of and greater temporal averaging in GUFM compared to POMME in particular in the southern hemisphere. The same is true for the CALS7K.2 results (Fig. 7). The average latitudinal dependence for the interval 1500 to 1950 AD is reasonably similar to that shown by GUFM for approximately the same time, while the scatter is significantly lower for the southern hemisphere. The CALS7K.2 model does not resolve the signature of the non-zonal structure seen by GUFM in the southern hemisphere.

In Fig. 8 we show the results averaged over the entire time interval spanned by CALS7K.2. The systematically low dipole moment persists in the southern hemisphere, but now the scatter is 

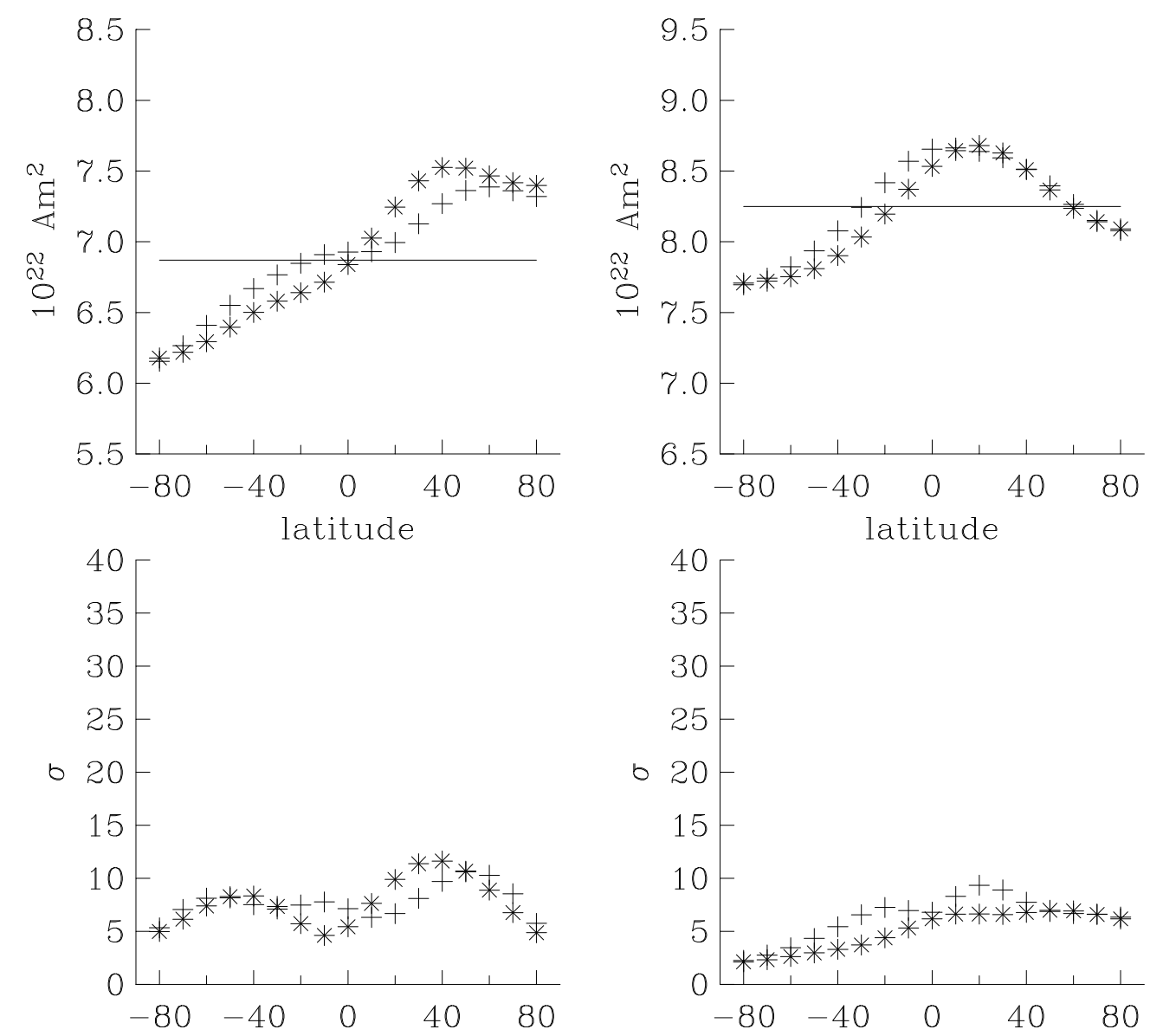

a)

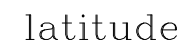

b)

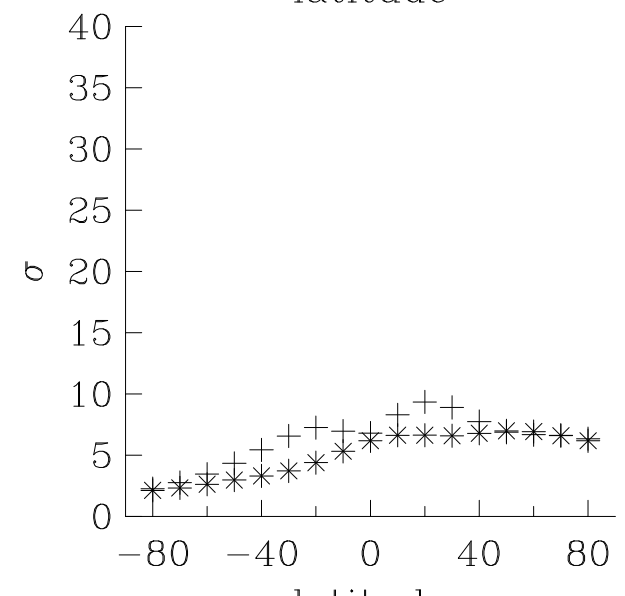

Fig. 7. VADMs $(+)$ and VDMs $(*)$ averaged on latitudinal circles and temporally from model CALS7K.2 (top) and their standard deviations in \% (bottom). a) Time interval $3000 \mathrm{BC}$ to $2000 \mathrm{BC}$, b) time interval $1500 \mathrm{AD}$ to $1950 \mathrm{AD}$. The solid line is the averaged SHA dipole moment $M$ for each interval.

essentially equal at all latitudes.

\section{Conclusions}

We have presented a new estimate for the geomagnetic dipole moment based on spherical harmonic analysis for the time period $5000 \mathrm{BC}$ to $1950 \mathrm{AD}$, and compared it with estimates computed by other techniques. We find that the existing estimates of the dipole moment based on paleomagnetic VADMs (4) are too high by about $19 \%$. Two factors contribute to this bias: data quality and contributions from the non-dipole field that are not elim- 

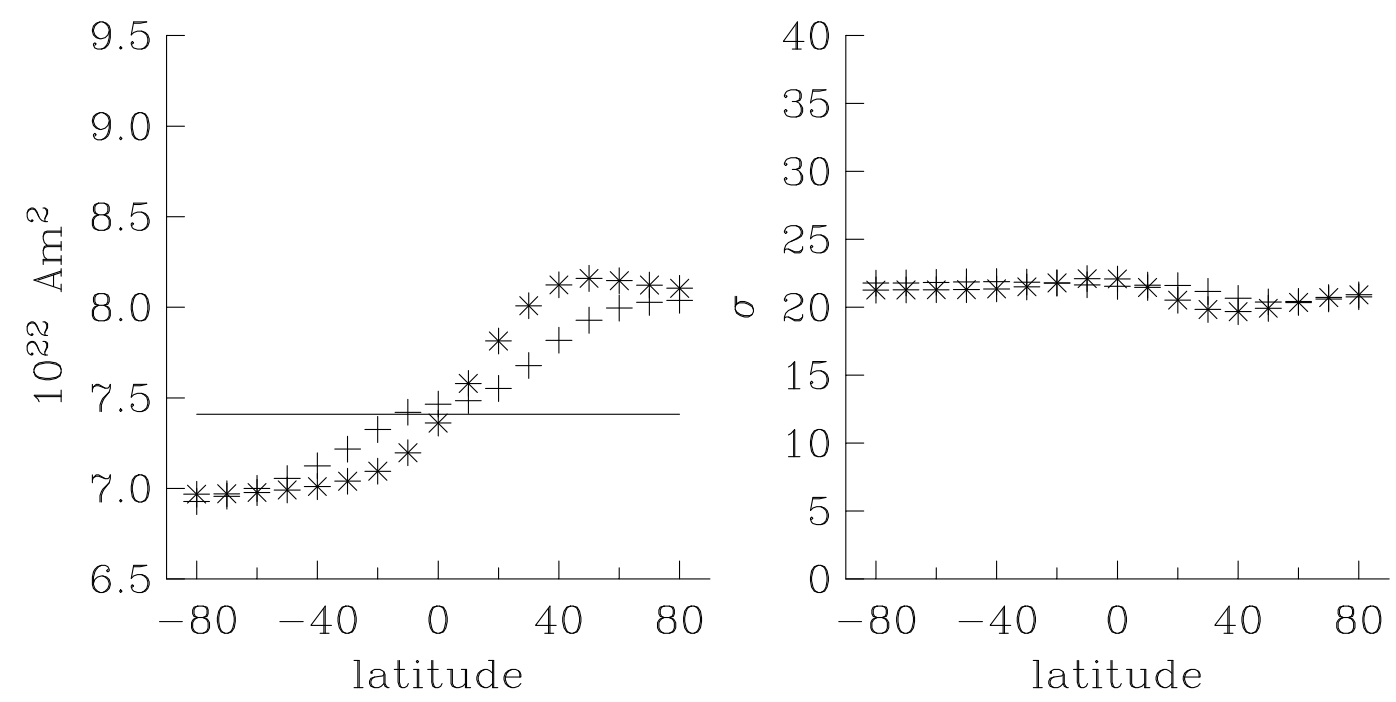

Fig. 8. VADMs $(+)$ and VDMs $(*)$ averaged over latitudinal circles and temporally over the time interval $5000 \mathrm{BC}$ to $1950 \mathrm{AD}$ from CALS7K.2 (left) and their standard deviations in \% (bottom). The solid line is the averaged SHA dipole moment $M$.

inated despite the averaging of VADMs over 500 or 1000 year time windows. The latter is aggravated by geographic biases in sampling.

In CALS7K.2 3\% of the paleointensity data were rejected because they were incompatible with data from the surrounding region and could not be fit by a reasonably simple spherical harmonic model. These are unlikely to be representative of the true dipole moment, but their exclusion has a minimal effect, reducing the average VADM over the $7 \mathrm{kyr}$ period from 9.27 to $9.24 \cdot 10^{22}$ $\mathrm{Am}^{2}$. The average data uncertainties are large $(11.1 \mu \mathrm{T})$, and the remaining 3092 data have been fit to this tolerance level by CALS7K.2. The intensity residuals show a slight bias: the data are under-fit by $3.6 \mu \mathrm{T}$ on average. This misfit cannot be accommodated in the dipole part of the field as justified above, but it explains the the $9.6 \%$ average difference between the VADMs $A_{m}$ predicted from CALS7K.2 and the raw averages $A_{d}$ computed directly from the data.

The effects of geographic sampling bias were investigated using geomagnetic field models with various temporal resolutions. The present day model, POMME, shows a strong latitudinal dependence in both the average VADM and its dispersion. The disper- 
sion variations are related to non-zonal structure in the present field. Similar structure is seen in the 400 year average provided by GUFM, but the magnitudes of the variations are muted by the time averaging and lower resolution in the model. In a comparable calculation for CALSK7.1 for 1500-1950 AD and for 3000 BC to $2000 \mathrm{BC}$ we find that the average VADM remains strongly latitude dependent, but with diminished resolution of the structure seen in the historic data. The peak to peak variation can be as large as $20 \%$. The dispersion also varies with latitude over these time scales strongly suggesting that neither 500 nor even 1000 years is long enough to average out non-zonal structure in the field completely, even with a uniform, global data distribution. When similar calculations are carried out for the entire $7 \mathrm{kyr}$ the latitudinal variation in VADM persists, but the dispersion becomes quite uniform with latitude, and substantially larger than for the shorter time intervals. Thus over the $7 \mathrm{kyr}$ average we find no evidence for persistent hemispheric asymmetry in variability. It is unclear whether this is because there is none, or because the resolution of CALS7K.2 is inadequate to detect it. The spectra in Figure 2 imply that there is insufficient power in the non-dipole field contributions, compared with POMME and GUFM.

Fig. 9 shows a comparison between the SHA dipole moment of CALS7K.2, GUFM (1) and the discrete DGRF/IGRF models (see http://www.ngdc.noaa.gov/IAGA/vmod/igrf.html) for the overlapping time interval since 1832. Although GUFM goes back to 1590 we only show the later part, because the dipole moment has been extrapolated in that model for the early epochs where there are no historical intensity data. The results agree well only for the last two decades of CALS7K.2 (1930 to 1950), before that the spherical harmonic models based on direct field observations for 1840 -1950 are higher by up to $2.5 \%$ than from CALSK7.1 prior to 1950. Note that even for those epochs CASL7K.2 is based purely on archeo-/paleomagnetic results, no direct field observations were added. It is likely that the discrepancy is a direct result of the temporal resolution in CALS7K.2 which has little structure 


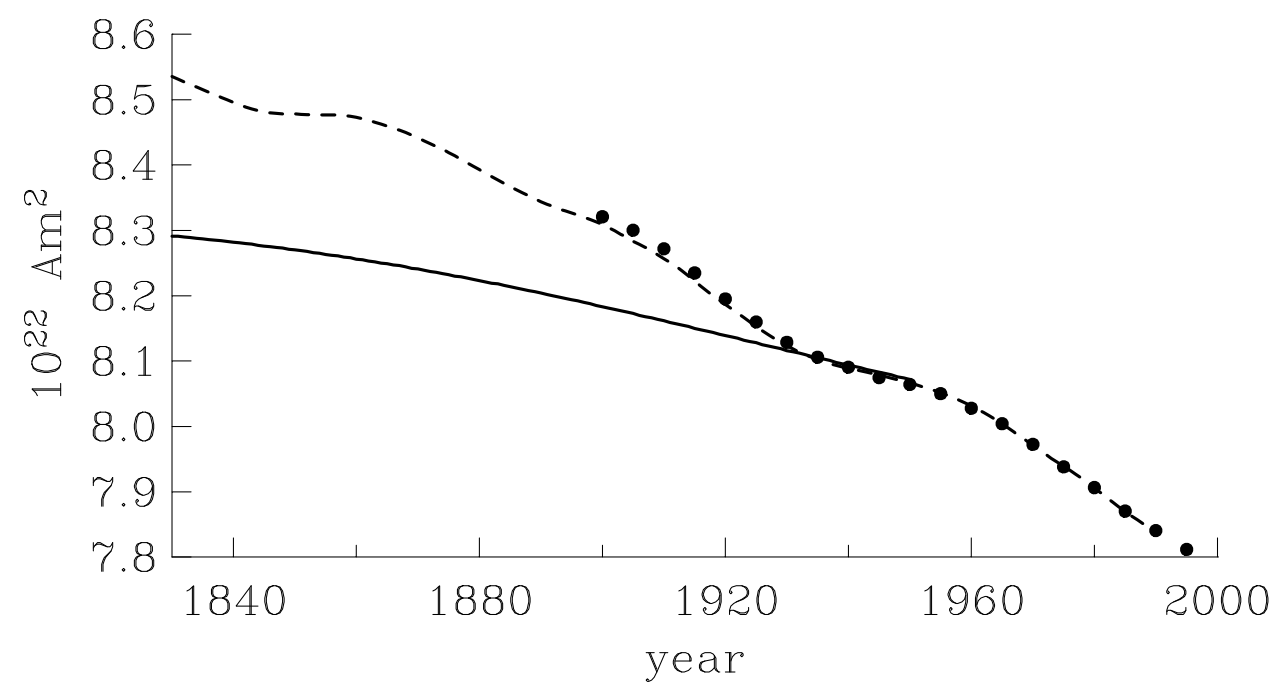

Fig. 9. Dipole moment of CALS7K.2 (solid line), GUFM (dashed line) and the discrete DGRF/IGRF models (dots)

on sub-centennial time scales.

The revisions to existing dipole moment estimates are substantial, but remember that they depend strongly on field geometry and data distribution, so they cannot be generalised to much earlier times. Our results indicate that the average dipole moment for $5000 \mathrm{BC}$ to $1950 \mathrm{AD}$ is $7.40 \cdot 10^{22} \mathrm{Am}^{2}$, slightly lower than the present value of $7.78 \cdot 10^{22} \mathrm{Am}^{2}$. This study suggests a peak value of about $9.5 \cdot 10^{22} \mathrm{Am}^{2}$ for the maximum of the dipole moment around $650 \mathrm{BC}$ and $320 \mathrm{AD}$ and minimum values below $5 \cdot 10^{22} \mathrm{Am}^{2}$ around $3920 \mathrm{BC}$ to the earliest times studied here. Previous VADM studies suggest that this was indeed a local minimum and the dipole moment was at about the level of 2000 BC for about two millennia before. The average decrease from the maximum value at $320 \mathrm{AD}$ on the revised curve gives about $25 \%$ in approximately 1700 years, a substantially lower rate than would be obtained for the past century. However, the rate of change is far from constant, and has varied from rates comparable to the current one to short intervening periods of increase. 


\section{Acknowledgements}

We wish to thank Agnès Genevey for some useful discussion. Jeff Love and an anonymous reviewer provided constructive reviews to improve the manuscript. CC acknowledges support from the US NSF grant EAR 0112290.

\section{References}

[1] A. Jackson, A. R. T. Jonkers, M. R. Walker, Four centuries of geomagnetic secular variation from historical records, Phil. Trans. R. Soc. Lond. A 358 (2000) 957-990.

[2] J.-P. Valet, Time variations in geomagnetic intensity, Rev. Geophys. 41 (2003) doi:10.1029/2001RG000104.

[3] M. W. McElhinny, W. E. Senanayake, Variations in the geomagnetic dipole: I. The past 50000 years, J. Geomag. Geoelectr. 34 (1982) 39-51.

[4] S. Yang, H. Odah, J. Shaw, Variations in the geomagnetic dipole moment over the last 12000 years, Geophys. J. Int. 140 (2000) 158-162.

[5] A. Genevey, Y. Gallet, M. Korte, C. G. Constable, A new archeointensity database for the past 10 millennia, in preparation (2004).

[6] M. Korte, C. G. Constable, Continuous geomagnetic field models for the past 7 millennia II: CALS7K, Geochem., Geophys., Geosys., doi:10.1029/2004GC000801, in press (2004).

[7] G. Hulot, A. Khokhlov, J. L. LeMouël, Uniqueness of mainly dipolar magnetic fields recovered from directional data, Geophys. J. Int. 129 (1997) 347-354.

[8] R. T. Merrill, M. W. McElhinny, P. L. McFadden, The Magnetic Field of the Earth, Academic Press, 1996.

[9] M. Korte, A. Genevey, C. F. Constable, U. Frank, E. Schnepp, Continuous geomagnetic field models for the past 7 millennia I: a new global data compilation, Geochem., 
Geophys., Geosys., doi:10.1029/2004GC000800, in press (2004).

[10] P. J. Smith, The intensity of the ancient geomagnetic field: a review and analysis, Geophys. J. R. Astron. Soc. 12 (1967) 321-362.

[11] C. E. Barton, R. T. Merrill, M. F. Barbetti, Intensity of the Earth's magnetic field over the last 10000 years, Phys. Earth Planet. Inter. 20 (1979) 96-110.

[12] S. Maus, H. Lühr, G. Balasis, M. Rother, M. Mandea, Introducing POMME, the POtsdam Magnetic Model of the Earth, in: C. Reigber, H. Lühr, P. Schwintzer, J. Wickert (Eds.), Earth Observation with CHAMP - Results from Three Years in Orbit, Springer Verlag, Berlin, 2004, pp. 293298. 